\title{
On the theory of relativistic collapse and relativistic explosion. 2. Quasistable states as glowsars and bursts at recollapse.
}

\author{
Zahid Zakir ${ }^{1}$
}

\begin{abstract}
At the gravitational collapse of a star the local temperatures in internal layers grow more rapidly than on surface, while the last one grows on world time exponentially. For this reason it is probable formation not a frozar, but an optimistic scenario of the collapse when matter of a star rapidly transfers into the radiation-dominated state with stabilization or further expansion (anticollapse). As a result, at stabilization the star can long on world time be in a hot phase (on local temperature). The semi-frozen relativistic objects in such quasistable phase release highly reddened quasirelict radiation and it is proposed to name this new class of objects as glowsars (glow star). Most of matter of glowsar concentrates near its surface in a radiation-dominated state. At defrosting of the glowsar its expansion at a final stage happens exponentially rapidly (the relativistic explosion) and the object is observed as the relativistic supernova or hypernova. Observable examples of the relativistic explosion, or anticollapse, are, perhaps, the Big Bang and some explosions in astrophysics with huge energy release, do not explained by known processes. Some idealized models and numerical simulations testify that at contracting of massive enough objects growing of pressure and temperature in internal layers can balance the gravitational pull and the explosive expansion is possible. The observable consequences of the predictions of GR about properties of the compact objects in glowsar state and the relativistic explosion are discussed.
\end{abstract}

PACS: 04.20.Dg; 04.70.-s; 97.60.-s, 98.54.-h

Key words: relativistic stars, collapse, frozars, glowsars, black holes, supernova, quasars

\section{Content}

Introduction

1. Big Bang as the relativistic explosion of the Universe .......................................................15

2. Quasistability and the relativistic explosion of supermassive stars ........................................16

3. Quasistability and the relativistic explosion of superdense stars ..........................................18

4. Glowsars and the relativistic super(hyper)novae in astrophysics ........................................19

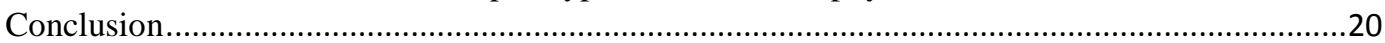

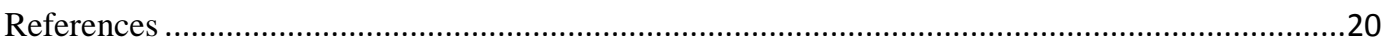

\section{Introduction}

In the first part of the paper [1] the structure of collapsing objects has been considered in the standard models of general relativity (GR) in the pessimistic scenario when the gravitational contracting dominates. It has been shown that here first of all freezes star's centre (in terms of astronomical world time), then middle layers and the surface freezes last of all to be beyond the gravitational radius $r_{g}=2 G M$. Thus in the

\footnotetext{
${ }^{1}$ Centre for Theoretical Physics and Astrophyics, Tashkent, Uzbekistan; zahidzakir@ theor-phys.org
} 
GR a collapsed star turns into a frozar state - a star with fully frozen internal structure where the world lines of particles timelike in entire volume and are almost parallel to the $t$ axis and among themselves [1-2].

Since on its surface the internal metric of any spherical non-rotating (and neutral) star is smoothly matched with the Schwarzschild metric, even at taking into account of a temperature and pressure, the surface asymptotically approaches $r_{g}$, never reaching it. However, non-linearity of the GR equations for the metric in the strong field can lead to such a combination of temperature and pressure of layers of a star that contracting can transfer into the pulsation or expansion.

Thus, in the present part of the paper we are beginning a preliminary study of such optimistic scenario when before a complete freezing of a star, pressure near its centre has time to be increased for balancing or exceeding the gravitational pull. Then the star does not freeze and turns into a quasistable phase with delayed rate of all processes, or can start expanding.

Thus, at the present stage there important not an equation of state, which is a phenomenological problem, but those general properties of such objects which follow from GR at the stabilization or recollapse of compact relativistic objects. In the paper two extreme cases - a supermassive object of small density and a superdense object of starlike mass - are considered. On these examples it is shown that the properties of opposite behavior of time and radial components of the metric on the surface and at centre are physically exhibited as the non-uniform growth of local temperatures and pressures. It then leads to rapid exponential growth of pressure at centre and further to pulsation or the relativistic explosion.

These objects at a relatively long time quasistable state, when the proper times are strongly dilated, turn into a hot phase in terms of local temperatures and emit radiation flows of very large redshift. Therefore the objects in such phase suitably to join into a new class and to call them as the glowsars (from «glow star»).

In the process of slow defrosting of the star and the subsequent exponentially rapid restoring of the proper time's rate, the expansion happens in two stages. At first the matter of central parts will concentrate near the surface, by forming a "thick shell" and transferring into the radiation-dominated phase. Then, at the second stage the surface will be expanded by explosive liberation of energy in the form of radiation and plasma with unusually powerful outburst. Radiation with the strong gravitational redshift becomes harder because of the kinematical violetshift. The objects in the relativistic explosion state can be exhibited as the relativistic supernovae or hypernovae.

In the paper the observable consequences of the GR theory of compact objects in the glowsar and the relativistic explosion states are considered also.

\section{Big Bang as the relativistic explosion of the Universe}

For such essentially important consequence of GR as transition into a hot phase with the subsequent relativistic explosion, even before detail consideration there is, although heuristic, but strong observational argument. From a simplified model of a star considered in the previous paper [2], as cutting from Freidmann's homogeneous world, logically follows that a hot phase at the contracting will be essential characteristic for destiny of a star also. From the GR model of the Universe, hot at the past, follows the model of the collapsing homogeneous star hot at the future.

Thus, if we consider the Big Bang (BB) as a recollapse after a hot phase of the Universe, this means that the same should be supposed for the relativistic star also - the 
collapse can lead to subsequent recollapse. This means that the star can go out from a hot phase by experiencing the relativistic explosion, which we can call further as the Local Bang (LB).

About a reality of the optimistic scenario of the relativistic collapse tell us following arguments and the observational facts:

(1) as an alternative to the optimistic phase of the collapse GR leaves only a frozar state, almost completely losing activity almost forever;

(2) observations confirm activity of compact objects and a possibility of the relativistic explosion or recollapse - except the Big Bang, other classes of powerful local explosions in astrophysics are known with rapid and unusually huge energy release, do not explained by usual or at least known mechanisms;

(3) the existence of an active phase of the collapse is admitted by the equations of GR, from the standard stellar models and some numerical simulations follows that at contracting of enough massive object the pressure near the centre grows more rapidly than at upper layers that can lead to a quasistability or expansion at first central regions and then higher layers.

All these testify that in GR from some critical mass relativistic explosion should be inevitable, otherwise would not be BB also. Therefore the problem not in, is there an explosion after a hot phase - basically it is admitted by GR and already observed as BB but in what of fundamental properties of the relativistic gravitation is responsible for that from some critical mass a star at the collapse to be fated to heating and expansion with pressure growing at the centre.

In following two sections on the example of two extreme cases - a supermassive object of small density and a superdense object of star-like mass - it is considered especially general relativistic property - opposite behavior of time and radial components of the metric at the surface and at centre. Physically this property is exhibited in the form of the nonuniform growth of local temperatures and pressures that further leads either to pulsation, or to exponential growth of pressure at centre and further explosion.

\section{Quasistability and the relativistic explosion of supermassive stars}

The metric of any spherical non-rotating (and neutral) star on its surface is smoothly matched with the Schwarzschild metric and consequently even at the account of temperature and pressure the star's surface asymptotically approaches $r_{g}$, never reaching it. In addition to this general property, from simplest idealized models of collapse in GR follows that at the strong contracting there are two properties of relativistic effects which do not depend on equations of state $[1,2]$ :

1) the proper time dilation coefficient on the surface is much less than at centre:

$$
e^{v\left(r_{b}\right) / 2} \ll e^{v(0) / 2},
$$

2) the radial contraction coefficient on the surface is much more than at centre (where there is no contraction):

$$
e^{\lambda\left(r_{b}\right) / 2} \gg e^{\lambda(0) / 2}=1
$$

To define, how these general properties will change the equations of state and dynamics of a star, at first we will consider a case of a supermassive star at which as a surface approaches $r_{g}$ a mean density still remains small and locally the ideal gas law remains valid: 


$$
p(r)=b \frac{T(r)}{\Delta V(r)}
$$

where $b=$ const and $p, T$ - pressure and temperature in a layer from $r-\Delta r$ up to $r$ with a local volume $\Delta V$.

As surface closely approaches $r_{g}$ the local temperature near the surface growths exponentially in world time $t$ :

$$
\frac{T\left[r_{b}(t)\right]}{T\left[r_{b}(0)\right]}=\frac{e^{v_{b}(0) / 2}}{e^{v_{b}(t) / 2}} \sim e^{t / 2 r_{g}} .
$$

The energy of a layer near the surface at falling from $r_{b}(0)$ to $r_{b}(t)$ in a local static frame will be subjected to the gravitational violetshift. If in the case of a dust the gravitational energy led only to the raising of the kinetic energy of the falling layer, in gas a part of this kinetic energy transforms into heat and the layer's temperature raises.

But, because of radial cuttings now in a layer from $r^{\prime}-\Delta r$ up to $r^{\prime}$ there will place in $e^{\lambda(r) / 2} / e^{\lambda\left(r^{\prime}\right) / 2}$ times more local standard radial "rods" and so the local volume element $\Delta V^{\prime}$ will increase:

$$
\frac{\Delta V\left(r^{\prime}\right)}{\Delta V(r)}=\frac{e^{\lambda\left(r^{\prime}\right) / 2}}{e^{\lambda(r) / 2}} \cdot \frac{r^{\prime 2}}{r^{2}},
$$

As a result, the particles of the gas with higher local mean energy will be distributed in a larger physical volume and, consequently, it follows from (3) - (5) that the local pressure near the surface does not depend on the relativistic factor:

$$
p_{b}\left(r^{\prime}\right)=b \frac{T_{b}\left(r^{\prime}\right)}{\Delta V_{b}\left(r^{\prime}\right)} \simeq \frac{r^{2}}{r^{\prime 2}} p_{b}(r),
$$

Let's consider what happens near centre of such star. As a surface approaches $r_{g}$, a local temperature near the centre raises anyway faster than on the surface. But now there are practically no radial contractions and in a layer $\Delta r$ near the centre a local volume element practically does not contain a relativistic factor and independent on $z$ :

$$
\frac{\Delta V_{c}\left(r^{\prime}\right)}{\Delta V_{c}(r)} \simeq \frac{r^{\prime 2}}{r^{2}} .
$$

As a result, particles of gas with higher mean local energy will be distributed in the same local volume and consequently at star's contraction according (3), (4) and (7) the local pressure near the centre contains the relativistic growing factor the same as for temperature:

$$
p_{c}\left(r^{\prime}\right)=b \frac{T_{c}\left(r^{\prime}\right)}{\Delta V_{c}\left(r^{\prime}\right)} \simeq\left(1+z^{\prime}\right) \frac{r^{2}}{r^{\prime 2}} p_{c}(r),
$$

Thus, in process of contracting of a supermassive star of small density happens exponentially rapid (in world time) raising of the local temperatures interior of the star larger than on the surface $T(r) \geq T_{b} \sim z \sim e^{t / r_{g}}$ which leads to the non-uniform raising of the local pressures. The local pressure near the surface changes insufficiently, whereas the local pressure at the centre raises exponentially rapidly (in world time) 
$p_{c} \sim z \sim e^{t / r_{g}}$ which at some stage leads to the stopping and expansion of internal layers of the star close to the surface with formation of a "thick shell". A further expansion of this shell then reveals as the relativistic explosion (burst).

Let's note a new and nontrivial relativistic effect which consists in that at the first stage there is an increasing of concentration of main part of star's mass-energy near its surface by formation of almost empty centre and a massive thick shell near surface.

Results of more detail numerical simulations of evolution of internal layers and the surface with restrictions on parameters of compact relativistic objects at the stopping of contracting and beginning of expansion will be presented in forthcoming publications.

\section{Quasistability and the relativistic explosion of superdense stars}

For superdense stars a hot phase arises for the same reasons as in a homogeneous cosmological model. As well as in cosmology, in the model of a contracting star at a hot phase to the total energy there contribute mainly the ultrarelativistic particles.

This allows us to accept the equation of state for the ultrarelativistic matter $p=\rho / 3$ as quite realistic for a hot stage of a stellar mass collapsing object practically at entire volume with the exception of a small "core" of radius $\delta r_{c}$ and a thin crust at the surface. Then the time component of the metric in this intermediate region is very simple:

$$
g_{00}(r)=\frac{r}{r_{b}}\left(1-\frac{2 G M\left(r_{b}\right)}{r_{b}}\right)=\frac{4}{7} \frac{r}{r_{b}}, \quad \delta r_{c}<r<r_{b}-\varepsilon,
$$

so that near the crust the time dilation coefficient $g_{00}^{-1 / 2}\left(r_{b}\right)$ is finite, while near to the core it is very large: $g_{00}^{-1 / 2}\left(\delta r_{c}\right) \sim g_{00}^{-1 / 2}\left(r_{b}\right) / \delta r_{c}^{1 / 2}$.

If at the first approximation we consider the contraction as adiabatic with reaching a thermodynamic equilibrium, a local temperature $T(t)$ will be distributed in the volume as:

$$
T(t)=g_{00}^{-1 / 2}(t) T_{0}=b \frac{T_{0}}{r^{1 / 2}} .
$$

On the surface it should be the Schwarzschild value of the metric that gives for the redshift's growth $z \sim e^{t / r_{g}}$ and this value is a minimum for inner layers of the star. Growth of the local temperature in internal layers stronger and consequently also will be proportional to $z$ on the surface:

$$
T(t)=\frac{T_{0}}{\sqrt{g_{00}(t)}} \sim e^{t / r_{g}} T_{0 b} \sim z T_{0 b} .
$$

At such exponentially rapid growth $z$ on the surface with the same growth of local temperatures, the matter into star transfers in the hot radiation-dominating phase and local values of pressure also grow exponentially:

$$
p(t) \sim T^{4}(t) \sim e^{4 t / r_{g}} p_{0} .
$$

Growth of local temperature opens channels of transformations of particles and also leads to phase transitions. Energy liberation at these reactions and phase transitions can lead to star's stabilization at some equilibrium value of parameters or to inverse expansion. 
At transition at first central, and then upper layers into quark phase with the energy releasing allowing to stop the contraction or leading to large ejections and explosion of the star, the layers with quark matter of $\mathrm{u}, \mathrm{d}$ quarks have approximately on $1 / 3$ higher energy, than the neutron matter. This energy gap allows us to consider a model of light quark stars as a probable for a part of compact objects heavier neutron stars.

In the literature there are many evidences that at numerical simulation with use the considered and other equations of state, such ball can transfer into an expansion state. Probably, one of the first it was noted still by Feynman [10]. More detail discussion of the results of numerical simulations will be presented in the forthcoming publications.

\section{Glowsars and the relativistic super(hyper)novae in astrophysics}

The exponential raising of the local pressure is characteristic for explosions and testifies that when an essential part of contraction the energy transforms into heat and then into radiation, the stopping of collapse by glowsar formation and/or expansion with hard radiation and outbursts not only become quite probable, but even may be inevitable.

Let's consider some general properties of glowsars which follow from their definition as a quasistable state at very large time dilation and high local temperatures:

(1) glowsars have sufficiently higher local temperatures and pressures at centre, than neutron stars and so radiate "quasirelict" photons and neutrino;

(2) in quasars and other active galaxy nuclei the gravitationally-frozen matter will be superdense only at a compact central region only, while a whole supermassive frozars and glowsars will have the complicated internal structure with matter in various phase states, including gravitationally-frozen layers also.

(3) there appears a new mechanism for a heavy elements synthesis which can be happen not only at supernovae, but also in glowsars also by essentially longer world time with subsequent outflowing at a relativistic explosion.

If an equation of state leads to revers of contraction with turning to a recollapse, then at a final stage, when the transition of a proper time's rate to the world time rate happens exponentially rapidly, glowsar transfers to the relativistic explosion state, becoming a relativistic supernova or hypernova. Defrosting of rate of proper time in the object with a radiation-dominated matter leads to the most powerful outburst of radiation from its surface expanded with a relativistic velocity.

Let's consider some properties of glowsars and relativistically exploding objects which follow from their basic distinctive features:

1. Energy losses because of various effects, bursts and explosions at internal phase transitions (also prolonged in time) can lead to their slow "evaporation" or (at a final stage) to rapid "defrosting" in the form of the relativistic supernova or hypernova with high violetshift due to high speed of expansion.

2. In galaxy nuclei and quasars the gravitationally-frozen matter will be superdense only in compact central parts, while a whole the supermassive frozars and glowsars will have a complex internal structure with matter in various phase states, including the gravitationally-frozen regions.

3. GR gives a new mechanism of explosion of supernovae and hypernovae with synthesis of heavy elements at the relativistic explosion.

4. Most powerful gamma ray bursts also can be related with the relativistic explosions. A distinctive feature of such processes is the fact that energy liberation will be comparable with a rest energy of matter and essentially exceeds an energy release at quark-hadron interactions (a nuclear synthesis or quark matter). 
5. A radiation flow from an expanding at relativistic velocities plasma (protons, nuclei) becomes hard due to a high violetshift. The strong gravitational redshift of photons reduces the estimations of power of such bursts and grows an estimation of a distance up to the source.

\section{Conclusion}

Thus, on the one hand, compact relativistic objects with hard equation of state, preventing the gravitational squeezing, because of dilating of proper times and all processes, turn into the states where the quasi-stabilization with stopping of contraction (glowsar), or recollapse with defrosting of the proper times (the relativistic explosion) can take place.

The observing types of the compact relativistic astrophysical objects (from stellar masses up to quasars) as a whole can be interpreted in the frameworks of GR as frozars, glowsar or relativistically exloded objects with various internal structure and nontrivial environment.

\section{References}

1. Zakir Z. Theor. Phys., Astrophys. and Cosmol., (2006) 1, 3, 42; (2007), 2, 1, 1; arXiv:0705.2585.

2. Zakir Z. (2012) Theor. Phys., Astrophys. and Cosmol., 7, 1, 1. 